\title{
Biologia reprodutiva e reprodução induzida de duas espécies de Characidae (Osteichthyes, Characiformes) da bacia do São Francisco, Minas Gerais, Brasil
}

\author{
Yoshimi Sato '; Edson V. Sampaio '; Nelsy Fenerich-Verani ${ }^{2}$ \& José R. Verani ${ }^{2}$ \\ ${ }^{1}$ Estação de Hidrobiologia e Piscicultura de Três Marias, CODEVASF. Caixa Postal 11, 39205-000 Três Marias, Minas Gerais, \\ Brasil. E-mail: cvsf3m@progressnet.com.br \\ ${ }^{2}$ Departamento de Hidrobiologia, Universidade Federal de São Carlos. Caixa Postal 676, 13565-905 São Carlos, São Paulo, \\ Brasil. E-mail: dnfv@power.ufscar.br
}

\begin{abstract}
Reproductive biology and induced breeding of two Characidae species (Osteichthyes, Characiformes) from the São Francisco River basin, Minas Gerais, Brazil. Astyanax bimaculatus (Linnaeus, 1758) and Tetragonopterus chalceus Spix \& Agassiz, 1829 are two important foraging species occurring at the São Francisco River basin. Specimens from both species were submitted to spawning induction through hypophysation, to study some reproductive features and to obtain subsidiary information for the definition of the artificial reproduction protocol. Males and females were treated with a single dose of crude carp pituitary extract $(6 \mathrm{mg} /$ $\mathrm{kg}$ ). In water at $26^{\circ} \mathrm{C}$, the extrusion of the oocytes was made 12,8 and 11 hours after the hypophisis injection, for A. bimaculatus and $T$. chalceus respectively. More than $70 \%$ of the females of both species showed a positive response to the treatment and mean egg fertilization rates were above $70 \%$. The eggs of $A$. bimaculatus and $T$. chalceus are yellow or medium brown, spherical, opaque, demersal, and slightly adhesive. The ova contained 4774 and 2563 eggs (mean values per gram) respectively. The embryonic development in both species was fast, lasting less than a day when water temperature was between 24 and $25^{\circ} \mathrm{C}$.

KEY WORDS. Astyanax bimaculatus; hypophysation; reproduction; Tetragonopterus chalceus.
\end{abstract}

RESUMO. Astyanax bimaculatus (Linnaeus, 1758) e Tetragonopterus chalceus Spix \& Agassiz, 1829 são duas importantes espécies de peixes forrageiros da bacia do São Francisco. Espécimes destas duas espécies foram submetidos à indução da desova através de hipofisação, para observação de aspectos reprodutivos e obtenção de subsídios para definição de protocolo de reprodução artificial. Machos e fêmeas receberam dose única de extrato bruto de hipófise de carpa comum ( $6 \mathrm{mg} / \mathrm{kg}$ de peixe). A extrusão de ovócitos foi realizada cerca de 12,8 e $11 \mathrm{~h}$ após a aplicação da injeção de hipófise, respectivamente para $A$. bimaculatus e $T$. chalceus, com a temperatura da água a $26^{\circ} \mathrm{C}$. Mais de $70 \%$ das fêmeas das duas espécies responderam positivamente ao tratamento e as taxas médias de fertilização dos ovos foram acima de $70 \%$. Os ovócitos de $A$. bimaculatus e $T$. chalceus são esféricos, opacos, demersais, levemente adesivos, de coloração amarela ou parda, e contendo em média respectivamente 4774 e 2563 ovos por grama de ova. O desenvolvimento embrionário nas duas espécies foi rápido, com duração de menos de um dia, estando a temperatura da água entre 24 e $25^{\circ} \mathrm{C}$.

PALAVRAS CHAVE. Astyanax bimaculatus; hipofisação; reprodução; Tetragonopterus chalceus.

Astyanax bimaculatus (Linnaeus, 1758) tem ampla distribuição geográfica na América do Sul (Lima et al. 2003) e comumente é denominada de piaba do rabo amarelo.

Astyanax bimaculatus pode atingir tamanho de $10,9 \mathrm{~cm} \mathrm{e}$ 42 g (Ihering \& AzEvedo 1936) e dependendo das condições ambientais, pode apresentar desova parcelada ou total (IHERING \& Azevedo 1936, Nomura 1975, Garutti 1989, Gennari Filho \& Braga 1996). As estimativas para o tamanho de maturação sexual da espécie variam de 7,8 a 10,4 cm de comprimento total (Nomura 1975, Agostinho et al. 1984, Santos et al.1991). Santos et al. (1991) estimaram em três anos a idade de primeira maturação gonadal em condições naturais e SiLvA et al. (1996) em quatro meses para a espécie em cativeiro. Sobre reprodução artificial de Astyanax têm-se as referências de Ihering \& Azevedo (1936) e Azevedo \& Canale (1938).

Tetragonopterus chalceus Spix \& Agassiz, 1829, ocorre nas bacias dos rios Amazonas, Orinoco e São Francisco e nas Guianas (ReIs 2003) e é conhecida por piaba rapadura.

Tetragonopterus chalceus da bacia amazônica atinge $10 \mathrm{~cm}$ de comprimento e desova duas vezes ao ano, em março e no- 
vembro (Magalhães 1931). Na represa de Três Marias (bacia do São Francisco) a espécie apresenta desova parcelada, onde a reprodução ocorre praticamente durante o ano todo; machos e fêmeas apresentam tamanho de primeira maturação gonadal de 4,8 e $6,3 \mathrm{~cm}$ de comprimento padrão, respectivamente (RicARDo et al. 1998). A única referência existente sobre reprodução artificial de Tetragonopterus é a de IherING \& WRIGHT (1935).

As duas espécies apresentam hábito alimentar onívoro, são de pequeno porte e importantes peixes forrageiros. São utilizadas como iscas na pesca esportiva, como petiscos, como peixes ornamentais e como peixes larvófagos no combate às larvas de pernilongos.

O objetivo do trabalho foi obter informações sobre a desova artificial e a biologia reprodutiva de $A$. bimaculatus e $T$. chalceus, ambas da bacia do rio São Francisco.

\section{MATERIAL E MÉTODOS}

O trabalho foi conduzido na Estação de Hidrobiologia e Piscicultura de Três Marias, da Companhia de Desenvolvimento dos Vales do São Francisco e do Parnaíba, Três Marias, Minas Gerais, no período de agosto de 2000 a fevereiro de 2001.

Reprodutores e matrizes de $A$. bimaculatus e $T$. chalceus foram capturados na represa de Três Marias (Três Marias, MG), bacia do rio São Francisco e estocados por um período de quatro a seis meses em gaiolas de tela plástica de $1,5 \mathrm{~m}^{3}$ e estas colocadas num viveiro de $200 \mathrm{~m}^{2}$, onde receberam como alimento ração granulada com $22 \%$ de proteína bruta (cerca de $2 \%$ da biomassa/dia, 5 dias/semana). Foram utilizados 40 machos e 25 fêmeas de $A$. bimaculatus e 40 machos e 28 fêmeas de $T$. chalceus.

A seleção dos reprodutores a hipofisação foi feita pela observação de características externas: fêmeas com o ventre abaulado, poro genital dilatado e avermelhado; machos com ventre fino, liberação de sêmen sob leve pressão do abdome e com espículas nos raios da nadadeira anal. Os reprodutores selecionados foram pesados, medidos e mantidos separados por sexo em gaiolas de tela plástica $0,224 \mathrm{~m}^{3}(0,8 \times 0,4 \times 0,7 \mathrm{~m})$ e estas colocadas dentro de tanque de alvenaria $2,4 \mathrm{~m}^{3}$ (3,0 x 1,0 x 0,8 m), com circulação constante de água, passando por um sistema de aquecimento, que a mantinha com temperatura de $26^{\circ} \mathrm{C}$ e com as seguintes características: oxigênio dissolvido $=5,5$ a 6,5 mg/L, $\mathrm{pH}=6$ a 7 , e condutividade elétrica $=60$ a $85 \mu \mathrm{S} / \mathrm{cm}$.

A indução da reprodução foi feita pelo método da hipofisação (IHERING \& AzEvedo 1934). Para provocar a liberação de gametas foi utilizado extrato bruto de hipófises de carpa comum Cyprinus carpio Linnaeus, 1758 (EBHC), injetado na cavidade celomática. Machos e fêmeas receberam dose única de $6 \mathrm{mg} \mathrm{EBHC/kg}$ de peixe.

A extrusão dos ovócitos e a coleta de sêmen foram executadas manualmente. A fertilização foi feita pelo método a "seco". O momento da desova foi estimado pelo cálculo: horas-grau à extrusão de ovócitos = tempo necessário para a desova a partir da aplicação da dose de EBHC (h) x temperatura da água $\left({ }^{\circ} \mathrm{C}\right)$.

Revista Brasileira de Zoologia 23 (1): 267-273, março 2006
A incubação dos ovos foi feita em incubadoras de $20 \mathrm{~L}$, em forma de funil, modelo Woynarovich (WoynARovich 1986), com circulação constante de água, cuja temperatura mantevese entre 24 e $25^{\circ} \mathrm{C}$. Nas incubadoras as vazões da água variaram de 0,5 a 1,0 L/min., e aumentou-se a vazão logo após o estádio de fechamento do blastóporo. As incubadoras receberam cerca de $20 \mathrm{~g}$ de ovos.

Das fêmeas obtiveram-se: comprimento total - CT (em $\mathrm{cm}$ ), peso corporal total - PT (em g), peso dos ovócitos extruídos (em g), número dos ovócitos extruídos/g de ova, peso dos ovócitos liberados e retidos nos ovários (ovócitos livres, mas remanescentes nas gônadas após a extrusão) (em g), diâmetros dos ovos não-hidratado e hidratado (em $\mu \mathrm{m}$ ), diâmetro do saco vitelino (em $\mu \mathrm{m})$, espaço perivitelino (em $\mu \mathrm{m})$, espessura do córion, incluindo, quando existente, a capa gelatinosa envolvente (em $\mu \mathrm{m})$, coloração e adesividade dos ovos, taxa de fertilização dos ovos (estimada a partir do estádio de fechamento do blastóporo) (em \%), peso das gônadas - PG (peso dos ovócitos extruídos + peso dos ovócitos liberados e retidos nos ovários (retirados após o sacrifício da fêmea depois da desova, em g), índice gonadossomático - IGS(PG.100/PT, em \%), fecundidade absoluta - FA (número de ovócitos extruídos + número de ovócitos liberados e retidos nos ovários), fertilidades inicial - FI (número de ovócitos extruídos), fertilidade final - FF (número de ovócitos fertilizados), horas-grau para a eclosão das larvas (temperatura da água, em ${ }^{\circ} \mathrm{C} x$ tempo desde a fertilização até a eclosão das larvas, em h), comprimento total das larvas recém eclodidas (em $\mu \mathrm{m}$ ), tipo de movimento da larva na coluna da água, presença ou não do órgão adesivo na cabeça da larva e tempo para formação das pós-larvas (em d). Fecundidade relativa, fertilidade inicial relativa e fertilidade final relativa foram estimadas em relação ao comprimento total e ao peso corporal das fêmeas.

As medidas dos diâmetros dos ovos (não hidratado e hidratado), da espessura do vitelo, da espessura do espaço perivitelino e da espessura do córion, foram efetuadas no estádio de blástula ou blástula alta (HisAOKA \& BATTLE 1958).

As terminologias de larva e pós-larva adotadas neste trabalho foram as de Woynarovich \& Horváth (1980).

Dos machos foram registrados: comprimento total (Lt, em $\mathrm{cm}$ ), peso corporal (Wt, em g), emissão de sons no período de reprodução (vocalização) e presença ou não de espículas nos raios da nadadeira anal.

\section{RESULTADOS}

Os espécimes de $A$. bimaculatus e $T$. chalceus estavam em condições de serem submetidos a hipofisação no período de outubro a fevereiro. Nestas espécies as fêmeas atingem porte maior que os machos.

Durante o período reprodutivo os machos das duas espécies não emitiram sons e apresentaram espículas nos raios da nadadeira anal, sendo que no caso de $T$. chalceus, na maioria das vezes de difícil percepção. 

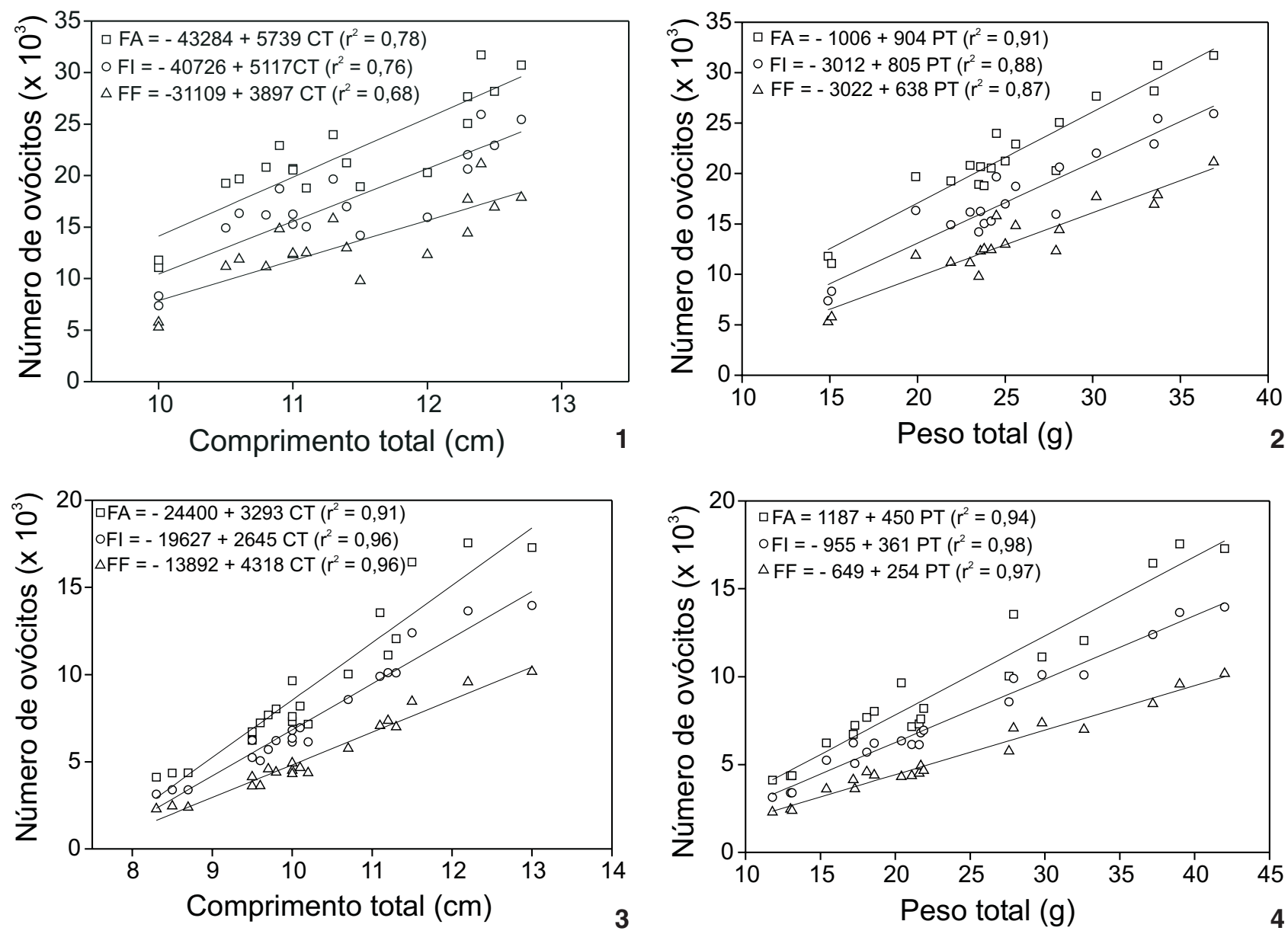

Figuras 1-4. Relações lineares da fecundidade absoluta (FA), fertilidade inicial (FI) e fertilidade final (FF) em funções das variações do comprimento total - CT (1) e do peso corporal total - PT (2), obtidas simultaneamente em 18 fêmeas de Astyanax bimaculatus e; em funções das variações do comprimento total - CT (3) e do peso corporal total - PT (4), obtidas simultaneamente em 20 fêmeas de Tetragonopterus chalceus, submetidas a hipofisação na Estação de Hidrobiologia e Piscicultura de Três Marias, no período de agosto de 2000 a fevereiro de 2001.

Cerca de $72 \%$ das fêmeas de $A$. bimaculatus e $71 \%$ de $T$. chalceus responderam ao tratamento, com liberação de ovócitos férteis.

A extrusão dos ovócitos foi provocada cerca de 12,8 h e $11 \mathrm{~h}$, respectivamente em $A$. bimaculatus e $T$. chalceus, após a aplicação da dose de EBHC, com a temperatura da água a $26^{\circ} \mathrm{C}$. As fêmeas das duas espécies sinalizaram o momento da desova, executando movimentos de um lado para o outro.

Os ovócitos de $A$. bimaculatus e T. chalceus são esféricos, opacos, demersais, levemente adesivos, com coloração amarela ou parda, sendo que somente nos da primeira espécie foi observada a presença de capa gelatinosa envolvente.

Os principais resultados obtidos estão registrados nas tabelas I e II.

As larvas das duas espécies apresentaram movimentos verticais na coluna da água e o órgão adesivo larval. O tempo de embriogênese foi relativamente rápido, sendo de cerca de $17 \mathrm{~h}$ em $A$. bimaculatus e de $20 \mathrm{~h}$ em $T$. chalceus, estando a água com temperatura entre 24 e $25^{\circ} \mathrm{C}$.

As relações entre fecundidade absoluta, fertilidade inicial e fertilidade final em funções da variação do peso corporal total e da variação do comprimento total das fêmeas estão indicadas nas figuras 1 e 2 para $A$. bimaculatus e nas figuras 3 e 4 para T. chalceus. Verificou-se que os valores destes parâmetros aumentaram proporcionalmente com o peso corporal total e com o comprimento total.

\section{DISCUSSÃO}

Machos de A. bimaculatus e de T. chalceus apresentaram espículas nos raios da nadadeira anal durante o período de re- 
Tabela I. Dados obtidos de exemplares de Astyanax bimaculatus, submetidos a hipofisação na Estação de Hidrobiologia e Piscicultura de Três Marias, no período de agosto de 2000 a fevereiro de 2001. (N) Número de observações, (EBHC) extrato bruto de hipófise de carpa comum, (DP) desvio-padrão, (CV) coeficiente de variação.

\begin{tabular}{|c|c|c|c|c|c|}
\hline Parâmetro & $\mathrm{N}$ & \multicolumn{2}{|c|}{ Média \pm DP } & $\mathrm{CV}$ & Amplitude \\
\hline \multicolumn{6}{|l|}{ Machos } \\
\hline Comprimento total $(\mathrm{cm})$ & 40 & $7,6 \pm$ & 1,3 & 16,9 & $5,8-$ \\
\hline Peso corporal total (g) & 40 & $4,7 \pm$ & 1,8 & 39,0 & $2,1-$ \\
\hline \multicolumn{6}{|l|}{ Fêmeas } \\
\hline Comprimento total $(\mathrm{cm})$ & 18 & $11,4 \pm$ & 0,8 & 7,5 & $10,0-$ \\
\hline Peso corporal total (g) & 18 & $25,3 \pm$ & 5,8 & 23,1 & $14,9-$ \\
\hline Horas grau à extrusão & 18 & $331 \pm$ & 7,0 & 2,2 & $320,0-\quad 340,0$ \\
\hline Índice gonadossomático (\%) & 18 & $18,0 \pm$ & 1,5 & 8,4 & $15,1-$ \\
\hline Peso dos ovócitos extruídos.100/PT (\%) & 18 & $14,2 \pm$ & 1,8 & 12,6 & $10,1-$ \\
\hline Número de ovócitos extruídos/g de ova & 18 & $4774 \pm$ & 92,0 & 1,9 & $4619,0-4925,0$ \\
\hline \multicolumn{6}{|l|}{ Medidas do ovo $(\mu \mathrm{m})$} \\
\hline Diâmetro do ovo não hidratado & $120^{*}$ & $1027 \pm$ & 31,0 & 3,0 & $977,0-1078,0$ \\
\hline Diâmetro do ovo hidratado & $120 *$ & $1143 \pm$ & 33,0 & 2,9 & $1065,0-1203,0$ \\
\hline Diâmetro do saco vitelino & $120 *$ & $642 \pm$ & 28,0 & 4,3 & $601,0-702,0$ \\
\hline Espaço perivitelino & $120^{*}$ & $212 \pm$ & 24,0 & 11,2 & $156,0-$ \\
\hline Espessura do córion & $120^{*}$ & $38 \pm$ & 8,0 & 22,3 & $30,0-$ \\
\hline Taxa de fertilização dos ovos (\%) & 18 & $75,3 \pm$ & 4,9 & 6,5 & $68,7-$ \\
\hline Fecundidade absoluta & 18 & \multicolumn{2}{|c|}{$21856 \pm 5529,0$} & 25,3 & $11086,0-31720,0$ \\
\hline Fertilidade inicial & 18 & \multicolumn{2}{|c|}{$17347 \pm 4990,0$} & 28,8 & $7369,0-25952,0$ \\
\hline Fertilidade final & 18 & \multicolumn{2}{|c|}{$13118 \pm 4000,0$} & 30,5 & $5291,0-21125,0$ \\
\hline FA relativa ( $\mathrm{n} \pm$ ovos/g de fêmea) & 18 & $861 \pm$ & 72,0 & 8,4 & $728,0-\quad 990,0$ \\
\hline FI relativa ( $\mathrm{n} \pm$ ovos extruídos/g de fêmea) & 18 & $678 \pm$ & 85,0 & 12,6 & $495,0-\quad 821,0$ \\
\hline FF relativa ( $\mathrm{n} \pm$ ovos viáveis/g de fêmea) & 18 & $511 \pm$ & 75,0 & 14,7 & $355,0-\quad 645,0$ \\
\hline FA relativa ( $\mathrm{n} \pm$ ovos/cm de fêmea) & 18 & $1905 \pm$ & 373,0 & 19,6 & $1109,0-2558,0$ \\
\hline FI relativa ( $\mathrm{n} \pm$ ovos extruídos/cm de fêmea) & 18 & $1509 \pm$ & 352,0 & 23,3 & $735,0-2093,0$ \\
\hline FF relativa ( $\mathrm{n} \pm$ ovos viáveis/cm de fêmea) & 18 & $1141 \pm$ & 292,0 & 25,6 & $529,0-1704,0$ \\
\hline Horas grau à eclosão & 18 & $414 \pm$ & 19,0 & 4,5 & $390,0-\quad 440,0$ \\
\hline CT da larva recém eclodida $(\mu \mathrm{m})$ & $120^{*}$ & $2284 \pm$ & 80,0 & 3,5 & $2129,0-2467,0$ \\
\hline
\end{tabular}

* Referente às medidas efetuadas para seis fêmeas (20 ovos ou larvas/fêmea).

produção. Este tipo de dimorfismo sexual aparece em vários representantes de Characidae como Salminus Spix \& Agassiz, 1829 (Ihering et al. 1928), Brycon Müller \& Troschel, 1844 (Kramer 1978) e Astyanax Baird \& Girard, 1854 (RACHow 1925 in Breder \& Rosen 1966), entre outros. Não foi observada emissão de sons pelos machos das duas espécies analisadas durante o período de reprodução, característica esta que é comum em representantes de Anostomidae, Curimatidae e Prochilodontidae (SATO et al. 2003a).

Fêmeas de A. bimaculatus e de T. chalceus sinalizaram o momento da desova, o que parece ser uma característica comum entre os representantes de Characiformes (SATO et al. 2003a).

O fato de mais de $70 \%$ das fêmeas de $A$. bimaculatus e de T. chalceus submetidas à hipofisação com a aplicação de uma dose de EBHC responderem positivamente ao experimento, indica que este procedimento pode ser considerado satisfatório na prática da reprodução artificial para estas duas espécies.

A extrusão de ovócitos de $A$. bimaculatus foi executada cerca de 331 horas-grau $(=12,8 \mathrm{~h})$ após a aplicação de dose única de EBHC e a de $T$. chalceus de 283 horas-grau (= $11 \mathrm{~h})$, com a temperatura da água a $26^{\circ} \mathrm{C}$. Ihering \& AzEvedo (1936), conseguiram desova de $A$. bimaculatus entre cinco e oito horas após a hipofisação e os reprodutores sinalizaram o momento da desova executando carrosséis.

À hidratação, os diâmetros dos ovos de A. bimaculatus passaram de $1,03 \mathrm{~mm}$ a 1,14 $\mathrm{mm}$, com aumento do volume da ordem de $36 \%$. As características de ovos de A. bimaculatus observadas aqui são semelhantes às descritas para esta espécie em outros trabalhos: IHERING \& AzEvedo (1936) verificaram que 
Tabela II. Dados obtidos de exemplares de Tetragonopterus chalceus, submetidos a hipofisação na Estação de Hidrobiologia e Piscicultura de Três Marias, no período de agosto de 2000 a fevereiro de 2001. (N) Número de observações, (EBHC) extrato bruto de hipófise de carpa comum, (DP) desvio-padrão, (CV) coeficiente de variação.

\begin{tabular}{|c|c|c|c|c|c|c|}
\hline Parâmetro & $\mathrm{N}$ & \multicolumn{2}{|c|}{ Média \pm DP } & $\mathrm{CV}$ & \multicolumn{2}{|c|}{ Amplitude } \\
\hline \multicolumn{7}{|l|}{ Machos } \\
\hline Comprimento total $(\mathrm{cm})$ & 40 & $8,6 \pm$ & 0,7 & 7,8 & $7,5-$ & 10,0 \\
\hline Peso corporal total (g) & 40 & $11,4 \pm$ & 3,0 & 26,1 & $7,0-$ & 18,6 \\
\hline \multicolumn{7}{|l|}{ Fêmeas } \\
\hline Comprimento total $(\mathrm{cm})$ & 20 & $10,2 \pm$ & 1,2 & 11,7 & $8,3-$ & 13,0 \\
\hline Peso corporal total (g) & 20 & $23,4 \pm$ & 8,9 & 38,1 & $11,8-$ & 42,0 \\
\hline Horas grau à extrusão & 20 & $283 \pm$ & 5,0 & 1,8 & $275-$ & 290,0 \\
\hline Índice gonadossomático (\%) & 20 & $16,0 \pm$ & 1,9 & 12,1 & $13,3-$ & 18,6 \\
\hline Peso dos ovócitos extruídos.100/PT (\%) & 20 & $12,8 \pm$ & 1,3 & & $10,7-$ & 15,1 \\
\hline Número de ovócitos extruídos/g de ova & 20 & $2463 \pm$ & 95,0 & 3,9 & $2217-$ & 2618,0 \\
\hline Medidas do ovo $(\mu \mathrm{m})$ & 20 & & & & & \\
\hline Diâmetro do ovo não hidratado & $200^{*}$ & $1254 \pm$ & 45,0 & 3,6 & $1143-$ & 1350,0 \\
\hline Diâmetro do ovo hidratado & $200^{*}$ & $2370 \pm$ & 76,0 & 3,2 & $2207-$ & 2545,0 \\
\hline Diâmetro do saco vitelino & $200^{*}$ & $813 \pm$ & 47,0 & 5,7 & $727-$ & 909,0 \\
\hline Espaço perivitelino & $200^{*}$ & $776 \pm$ & 42,0 & 5,4 & $686-$ & 880,0 \\
\hline Espessura do córion & $200^{*}$ & $2,4 \pm$ & 0,4 & 15,1 & $2,0-$ & 3,0 \\
\hline Taxa de fertilização dos ovos (\%) & 20 & $70,7 \pm$ & 3,1 & 4,4 & $66,1-$ & 80,2 \\
\hline Fecundidade absoluta & 20 & \multicolumn{2}{|c|}{$9335 \pm 4142,0$} & 44,4 & \multicolumn{2}{|c|}{$4105-17554,0$} \\
\hline Fertilidade inicial & 20 & \multicolumn{2}{|c|}{$7472 \pm 3247,0$} & 43,5 & \multicolumn{2}{|c|}{$3139-13967,0$} \\
\hline Fertilidade final & 20 & \multicolumn{2}{|c|}{$5274 \pm 2290,0$} & 43,4 & \multicolumn{2}{|c|}{$2282-10168,0$} \\
\hline FA relativa ( $\mathrm{n} \pm$ ovos/g de fêmea) & 20 & $393 \pm$ & 48,0 & 12,1 & $333-$ & 486,0 \\
\hline Fl relativa ( $\mathrm{n} \pm$ ovos extruídos/g de fêmea) & 20 & $314 \pm$ & 31,0 & 9,8 & $259-$ & 362,0 \\
\hline FF relativa ( $\mathrm{n} \pm$ ovos viáveis/g de fêmea) & 20 & $222 \pm$ & 22,0 & 9,7 & $181-$ & 253,0 \\
\hline FA relativa ( $\mathrm{n} \pm$ ovos/cm de fêmea) & 20 & $881 \pm$ & 290,0 & 32,9 & $495-$ & 1439,0 \\
\hline FI relativa ( $\mathrm{n} \pm$ ovos extruídos/cm de fêmea) & 20 & $705 \pm$ & 226,0 & 32,0 & $378-$ & 1119,0 \\
\hline FF relativa ( $\mathrm{n} \pm$ ovos viáveis/cm de fêmea) & 20 & $498 \pm$ & 158,0 & 31,7 & $273-$ & 784,0 \\
\hline Horas grau à eclosão & 20 & $483 \pm$ & 11,0 & 2,4 & $470-$ & 500,0 \\
\hline CT da larva recém eclodida $(\mu \mathrm{m})$ & $200 *$ & $2638 \pm$ & 67,0 & 2,6 & $2227-$ & 2788,0 \\
\hline
\end{tabular}

* Referente às medidas efetuadas para 10 fêmeas (20 ovos larvas/fêmea).

os ovos apresentavam camada pegajosa revestindo a cápsula e registraram 4150 ovócitos/g e AzEvedo \& CANALE (1938) encontraram ovos esverdeados e amarelados.

Tetragonopterus chalceus apresentou ovos opacos, demersais, esféricos, amarelos, levemente adesivos, contendo cerca de 2490 ovos/g de ova e sem a presença da capa gelatinosa envolvente. Com a hidratação os diâmetros dos ovos passaram de $1,25 \mathrm{~mm}$ a $2,37 \mathrm{~mm}$, com aumento do volume em cerca de $582 \%$. Segundo Winemiller (1989) ovócito maduro de $T$. argenteus Cuvier, 1816 apresenta diâmetro igual a $1 \mathrm{~mm}$.

O maior aumento de volume após a hidratação dos ovos de $T$. chalceus, em comparação aos de $A$. bimaculatus talvez seja em função da presença nesta última da capa gelatinosa envolvente.
O valor máximo de índice gonadossomático de fêmeas de $A$. bimaculatus foi de $20,6 \%$, próximo aos registrados na literatura para esta espécie (Tab. III). Tetragonopterus chalceus apresentou IGS máximo igual a 18,6\%.

Tabela III. Valores de índice gonadossomático (IGS) de fêmeas de Astyanax bimaculatus, durante o período reprodutivo.

\begin{tabular}{cl}
\hline IGS máximo (\%) & \multicolumn{1}{c}{ Autor } \\
\hline 32,8 & AzeVedo \& CANALE (1938) \\
21,0 & GarutTI (1989) \\
16,5 & VAzzoler (1996) \\
20,6 & Presente trabalho \\
\hline
\end{tabular}

Revista Brasileira de Zoologia 23 (1): 267-273, março 2006 
A fecundidade absoluta de A. bimaculatus variou de 11086 a 31720 ovócitos, estando dentro dos limites registrados para a espécie (Tab. IV). Tetragonopetrus chalceus apresentou fecundidade absoluta de 4105 a 17554 ovócitos.

Tabela IV. Valores de fecundidade absoluta de fêmeas de Astyanax bimaculatus..

\begin{tabular}{cl}
\hline Fecundidade absoluta & \multicolumn{1}{c}{ Autor } \\
\hline 39855 & IHERING \& AzEVEDo (1936) \\
$740-22452$ & Nomura (1975) \\
$4978-18040$ & GenNARI FilHo \& BraGA (1996) \\
$8300-32700$ & Santos et al. (1996) \\
$11085-31720$ & Presente trabalho \\
\hline
\end{tabular}

O valor de $\mathrm{r}^{2}$ foi melhor quando se relacionou a fecundidade absoluta com o peso corporal, do que com o comprimento total em $A$. bimaculatus (Figs 1 e 2 ) e foram semelhantes no caso de $T$. chalceus (Figs 3 e 4). Para esta espécie Gennari Filho \& Braga (1996), também encontraram $\mathrm{r}^{2}$ maior para a relação com o peso corporal, ao passo que NomUra (1975) encontrou valor melhor quando se relacionou com o comprimento total. SANTos et al. (1996) não encontraram diferença quando se relacionou fecundidade absoluta com o comprimento padrão e peso corporal.

A eclosão das larvas de $A$. bimaculatus ocorreu em média a 410 horas-grau (tempo de duração $=17 \mathrm{~h}$ ) e as de $T$. chalceus a 499 horas-grau (tempo de duração $=20$ h) após a fertilização dos ovos, estando a temperatura da água entre 24 e $25^{\circ} \mathrm{C}$, e as larvas mediram em média 2,28 mm e 2,64 mm, respectivamente. IHERING \& AzEvEDo (1936) observaram eclosão das larvas de A. bimaculatus depois de $36 \mathrm{~h}\left(18^{\circ} \mathrm{C}\right), 18 \mathrm{~h}\left(23^{\circ} \mathrm{C}\right)$ e $12 \mathrm{~h}\left(28^{\circ} \mathrm{C}\right)$ e as larvas recém eclodidas mediram de 2,54 a 2,61 mm de comprimento.

As larvas das duas espécies estudadas apresentaram movimentos verticais na coluna da água e transformaram em póslarvas, 3,5 a 4 dias após a eclosão. Notou-se a presença de órgão adesivo na cabeça das larvas, estrutura esta anteriormente observada por IHERING \& AzEvedo (1936) em A. bimaculatus, cuja duração se restringiu a pouco mais de 36 horas, com a formação de pós-larvas depois de 2,5 a 3 dias. A finalidade do órgão adesivo larval é a de permitir a fixação das larvas principalmente em vegetais.

As duas espécies estudadas apresentaram características semelhantes: ovo adesivo e pequeno, embriogênese de curta duração, órgão adesivo larval, número elevado de ovos por grama de ovário, alta fecundidade por peso corporal, índice gonadossomático elevado, pequeno diâmetro do ovo não hidratado, larva recém eclodida pequena e com pequeno comprimento total do peixe.

Baseado nas características acima referidas, SATO et al. (2003b) consideraram as duas espécies como pertencentes ao grupo dos peixes de estratégia reprodutiva oportunista (tendência $r$-estrategista), seguindo as proposições de Winemiller (1989) e Winemiller \& Rose (1992) ou ao grupo (guilda) dos fitolitófilos não guardadores, que desovam em áreas com plantas, não obrigatoriamente, seguindo a classificação proposta por BALON (1985).

\section{AGRADECIMENTOS}

Ao Convênio CEMIG/CODEVASF (CV 09500 - 0012/ 00), pelas facilidades na execução do trabalho.

\section{REFERÊNCIAS BIBLIOGRÁFICAS}

Agostinho, C.A.; S.L. Molinari; A.A. Agostinho \& J.R. Verani. 1984. Ciclo reprodutivo e primeira maturação sexual de fêmeas do lambari, Astyanax bimaculatus (L.) (Osteichthyes Characidae) do rio Ivaí, Estado do Paraná. Revista Brasileira de Biologia, Rio de Janeiro, 44 (1): 31-36.

Azevedo, P. \& L. Canale. 1938. L. A hipófise e sua ação nas gônadas dos peixes neotrópicos. Arquivos do Instituto Biológico, São Paulo, 9: 165-186.

BALON, E.K. 1985. Early life histories of fishes: new development, ecological and evolutionary perspectives. Dordrecht, W. Junk Publishers, 280p.

BREDER JR., C.M. \& D.E. Rosen. 1966. Modes of reproduction in fishes. New York, Natural History Press, 941p.

GarutTI, V. 1989. Contribuição ao conhecimento reprodutivo de Astyanax bimaculatus (Ostariophysi, Characidae), em cursos de água da bacia do rio Paraná. Revista Brasileira de Biologia, Rio de Janeiro, 49 (2): 489-495.

Gennari Filho, O. \& F.M.S. Braga. 1996. Fecundidade e desova de Astyanax bimaculatus e A. schubarti (Characidae, Tetragonopterinae) na represa de Barra Bonita, rio Piracicaba (SP). Revista UNIMAR, Maringá, 18 (2): 241-254.

Hisaoka, K.K. \& H.I. Battle. 1958. The normal development of the zebrafish, Brachydanio rerio (Hamilton-Buchanan). Journal of Morphology, New York, 102: 311-328.

IHERING, R. vON \& P. AzEvedo. 1934. A curimatã dos açudes nordestinos (Prochilodus argenteus). Arquivos do Instituto Biológico, São Paulo, 5: 143-184.

Ihering, R. von \& P. AzEvedo. 1936. As piabas dos açudes nordestinos (Characidae, Tetragonopterinae). Arquivos do Instituto Biológico, São Paulo, 7: 75-110.

Ihering, R. von; J.C. Barros \& N. Planet. 1928. Os óvulos e a desova dos peixes d'água doce do Brasil. Boletim Biológico, São Paulo, 14: 97-109.

Ihering, R. von \& S. Wright. 1935. Fisheries investigations in Northeast Brazil. Transactions of the American Fisheries Society, Washington, 35: 267-271.

KRAMER, D.L. 1978. Terrestrial group spawning of Brycon petrosus (Pisces: Characidae) in Panama. Copeia, Lawrence, 1978 (3): 536-537.

Lima, F.C.T.; L.R. Malabarba; P.A. BuckuP; J.F.P. Silva; R.P. Vari; A. 
Harold; R. Benine; O.T. Oyakana; C.S. Pavanelli; N.A. Menezes; C.A.S. Lucena; M.C.S.L. Malabarba; Z.M.S. Lucena; R.E. Reis; F. Langeani; L. Cassati; V.A. Bertaco; C. Moreira \& P.H.F. LUCINDA. 2003. Genera Incertae Sedis in Characidae, p.106169. In: R.E. Reis; S.O. Kullander \& C.J. Ferraris Jr. (Eds). Check list of the freshwater fishes of South and Central America. Porto Alegre, EDIPUCRS, 742p.

Magalhães, A.C. 1931. Monographia brazileira de peixes fluviaes. São Paulo, Graphicars, 260p.

Nomura, H. 1975. Fecundidade, maturação sexual e índice gônado-somático de lambari do gênero Astyanax Baird \& Girard, 1854 (Osteichthyes, Characidae), relacionados com fatôres ambientais. Revista Brasileira de Biologia, Rio de Janeiro, 35 (4): 775-798.

ReIs, R.E. 2003. Subfamily Tetragonopterinae, p.212. In: R.E. Reis; S.O. Kullander \& C.J. Ferraris JR. (Orgs). Check list of the freshwater fishes of South and Central America. Porto Alegre, EDIPUCRS, 742p.

Ricardo, M.C.P.; E. Rizzo; Y. SATo \& N. Bazzoli.. 1998. Análise histológica da reprodução de Tetragonopterus chalceus Agassiz, 1829 (Pisces: Characidae) na represa de Três Marias, Minas Gerais. Bios, Belo Horizonte, 6 (6): 113-120.

Santos, R.A.; E.C. Campos, E.C.; J.J.C. Camara \& J. Mandelli Júnior. 1991. Curvas de maturação gonadal e crescimento de fêmeas de tambiú, Astyanax bimaculatus Linnaeus, 1758 (Characiformes, Characidae), na represa de Ibitinga, Estado de São Paulo, Brasil. Boletim do Instituto de Pesca, São Paulo, 18: 1-11.

Santos, G.B.; L.N.V. Barros \& M.U. Menezes. 1996. Fecundity of the Astyanax bimaculatus (Reinhardt, 1874) (Teleostei, Characidae) from Upper Rio São Francisco basin, Brazil. Arquivo
Brasileiro de Medicina Veterinária e Zootecnia, Belo Horizonte, 48 (Supl. 1): 101-108.

Sato, Y.; N. Fenerich-Verani \& H.P. Godinho. 2003a. Reprodução induzida de peixes da bacia do São Francisco, p.275-289. In: H.P. Godinho \& A.L. Godinho (Eds). Águas, peixes e pescadores do São Francisco das Minas Gerais. Belo Horizonte, PUC Minas, 468p.

Sato, Y.; N. Fenerich-Verani; A.P.O. Nuñer; H.P. Godinho \& J.R. Verani. 2003b. Padrões reprodutivos de peixes da bacia do São Francisco, p.229-274. In: H.P. GodinHo, A.L. Godinho (Eds). Águas, peixes e pescadores do São Francisco das Minas Gerais. Belo Horizonte, PUC Minas, 468p.

Silva, J.V.; D.R. Andrade \& W.Y. Okano. 1996. Desenvolvimento sexual e crescimento de lambaris - tambiú, Astyanax bimaculatus Linnaeus, 1758 submetidos a diferentes tipos de alimentação. Arquivo de Medicina Veterinária e Zootecnia, Belo Horizonte, 48 (Supl. 1): 47-54.

VAZzoler, A.E.A. 1996. Biologia da reprodução de peixes teleósteos: teoria e prática. Maringá, EDUEM, SBI, 169p.

WinemiLler, K.O. 1989. Patterns of variation in life history among South American fishes in seasonal environments. Oecologia, Paris, 81: 225-241.

Winemiller, K.O. \& K.A. Rose. 1992. Patterns of life-history diversification in North American fishes: implications for population regulation. Canadian Journal of Fisheries and Aquatic Sciences, Ottawa, 49 (10): 2196-2218.

Woynarovich, E. 1986. Tambaqui e pirapitinga: propagação artificial e criação de alevinos. Brasília, CODEVASF, 68p.

Woynaroch. E. \& L. Horváth. 1980. The artificial propagation of warm-water finfishes - a manual for extension. FAO Fisheries Technical Paper, Rome, 201: 1-183.

Recebido em 14.VI.2005; aceito em 03.III.2006. 